\title{
Results of Optical Signals Analyses Emitted by Electrical Discharges Recorded with UV Camera
}

\author{
P. Frącz*, T. Boczar, S. Borucki, A. Cichó́ and D. ZmarzŁy \\ Faculty of Electrical Engineering, Automatic Control and Computer Science \\ Opole University of Technology, Prószkowska 76, 45-758 Opole, Poland
}

\begin{abstract}
In the article results of measurements and analyses of the optical signals emitted by electrical surface partial discharges occurring on bushing and post insulators, recorded with UV camera during tests under laboratory conditions are presented. The carried out research was to determine the effect of the distance between the insulator's electrodes and the voltage value on the number of partial discharges.
\end{abstract}

PACS: 79.60.Bm, 42.79.Pw, 52.80.Hc

\section{Introduction}

One of the fundamental problems of insulation systems being used in electrical equipment and high voltage transmission lines is the aging process that occurs during their operation, and deterioration of insulating properties caused by it. Impurities that accumulate on the surface of the high voltage insulators decrease their insulating abilities. The presence of dirt, as well as high level of humidity cause conductive path formations, which are the source of partial discharges (PD) [1]. The quantity, amplitude and intensity of the $\mathrm{PD}$, which can be generated in the high-voltage power engineering devices are critical values, when evaluating and determining the insulation quality. Their occurrence can have a direct impact on the stability and proper operation of the power engineering system because they can contribute to a failure, and in extreme cases, cause power outages. This leads to constant development of different techniques to improve the methods being used for detection, location and PD intensity measurement [2-11]. In addition to the conventional methods of PD measuring (electrical methods, $\mathrm{HF} / \mathrm{VHF}$, UHF), non-destructive alternative methods for diagnosis of insulation systems such as: optical methods, mechanical (acoustic and opto-acoustic), and chemical, are more commonly used.

The aim of the research work, whose results are presented in this article, was to record and perform the analysis of the optical radiation generated by partial surface discharges (SPD) that was carried out on the models of bushing and post insulators.

\section{Scope of analyses considered in this paper}

The performed measurement range of the ultraviolet (UV) radiation emitted by the $\mathrm{PD}$, being generated on the examined bushing and post insulators' models, whose

\footnotetext{
* corresponding author; e-mail: p.fracz@po.opole.pl
}

results are presented in this article, has covered the impact assessment on the obtained results by changing the following factors:

- The distance between the electrodes of the given insulator. The measurements were performed for the four examined models of insulators by analysing the distances in the range from 1 to $12 \mathrm{~cm}$, with gradation of $1 \mathrm{~cm}$. The same aluminium input with diameter of $9.9 \mathrm{~mm}$ was used for bushing insulators (model I and II). During the tests, the supply voltage was adjusted, in the range from 0 to $0.99 U_{\mathrm{p}}$ (breakdown voltage), for each distance being analysed.

- Values of the supply voltage applied to an aluminium clamp, for the constant distance between the electrodes equals to $10 \mathrm{~cm}$. The recording of the $\mathrm{PD}$ counts was made by increasing the supply voltage in the range from 0 to $0.99 U_{\mathrm{p}}$. In addition, the impact of the diameter of the aluminium inlay for bushing insulators (model I and II) was examined, analysing the inlays of a diameter of $9.9 \mathrm{~mm}$ and $4.9 \mathrm{~mm}$, to compare.

In order to undertake a comparative analysis of the obtained relationships, a research, being related to the assessment of the impact of the above-mentioned factors on the recorded results, was performed for the same UV camera parameters, such as: relative sensitivity of 90 , for the maximum size of window counts, and without digital zoom (gain) of the recorded occurrences. The measurements were carried out at fixed distance of $2.3 \mathrm{~m}$ between the lens of the UV camera and the place of the PD generation.

\section{Methodology of the experiment and the UV camera specification}

For measurement of the optical radiation being emitted by the SPD that are generated in the post insulator modelling system, professional camera UVollé by OFIL Systems was used, designed to monitor electric power networks, either the medium, high and highest voltage. The Uvollé camera has the following parameters: spectral range (250-280) nm, sensitivity in the UV range: 
$3 \times 10 \mathrm{~W} / \mathrm{cm}^{2}$, the minimum sensitivity of illumination: 1 lux, solid angle with an accuracy more than 1 milliradian. The camera uses detectors that simultaneously record images generated at the $\mathrm{PD}$ insulating systems in two bands: UV (in the range from $250 \mathrm{~nm}$ to $280 \mathrm{~nm}$ ) and visible. The measurement principle is based on counting the number of $\mathrm{PD}$ using an UV radiation sensitive matrix (number of pixels active in the UV band), and imposing the image that was obtained in this way, on the visible picture of the tested electrical apparatus, which is obtained by using standard CCD matrix that is sensitive on visible light. After one image is imposed to another, the precise image of PD intensity is obtained, while the remaining part of the picture represents the picture in visible light. The lens and optical filter that are used in the camera have been optimized to illustrate the PD effect and its surroundings in the visible light. Thus, the detection - point location of occurrences and PD intensity measurement - is possible. Additionally, the UVolle camera is equipped with noise reduction algorithm, by which the occasional single UV signals are being eliminated. During measurements, the recorded pictures were displayed directly on a colour LCD screen, and then recorded on the SD memory card as images.

An insulator model, which was built as a porcelain tube filled with transformer's oil, was used to generate the SPD. The examined insulator model was powered by a test system, which allows to smooth voltage control in the range from 0 to $60 \mathrm{kV}$. During all measurements constant distance between the camera and the bushing and post insulator models was kept. The recorded data were processed, and then analysed using calculation procedures developed in Matlab. A detailed description of the test system and insulators models is presented in the study paper [11].

\section{The analysis of the results obtained}

For comparison, in Fig. 1 there were given some characteristics, which illustrate changes in the number of PD counts as a function of supply voltage. Dependence graphs were designated for all of the insulator models, at the same distance between the electrodes, equal to $10 \mathrm{~cm}$, and for the same values of the parameters specified in Sect. 2. Additionally, characteristics obtained for bushing insulators (model I and II), for the inlay of a diameter of $4.9 \mathrm{~mm}$, were presented.

For comparison, Fig. 2 shows examples of characteristics describing the correlation between the number of PD counts recorded with UV camera and the distance between the electrodes. Separately, for the four examined models graphs were determined to show the correlation for the considered distance values, ranging from 1 to $12 \mathrm{~cm}$. For each of the examined distance, the value of the supply voltage was adjusted from 0 to $0.99 U_{\mathrm{p}}$.

Based on the results obtained in the previous research tests, whose outcomes are presented in the article [2], no influence of the voltage regulation method (decreasing,

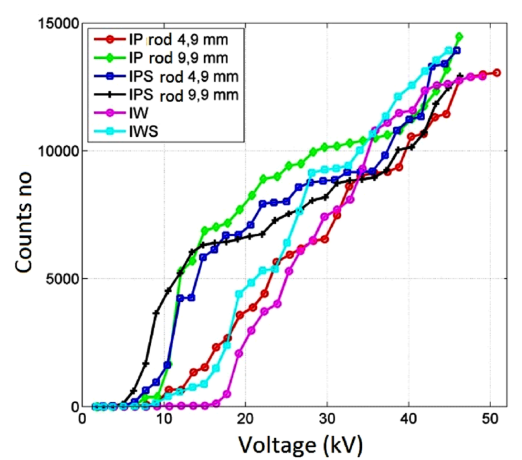

Fig. 1. Correlation between the number of PD counts and the supply voltage value, comparatively determined for the examined insulator models, for constants: distance between the electrodes, sensitivity and gain.

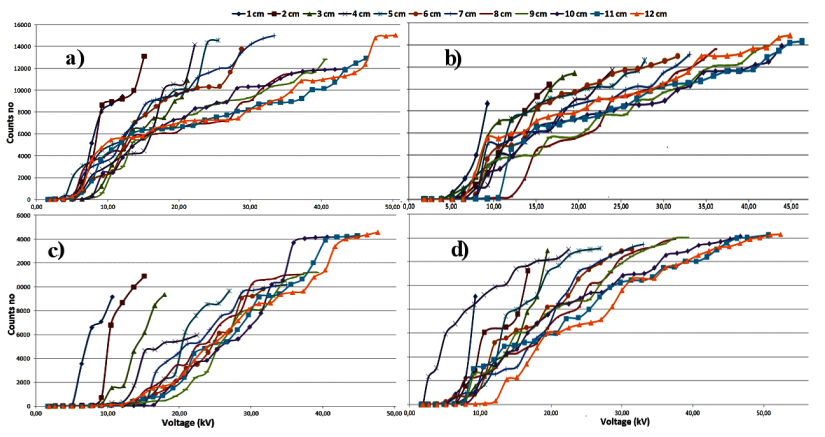

Fig. 2. Correlation between the number of PD counts for the distance between electrodes ranging from 1 to $12 \mathrm{~cm}$, and (a) bushing insulator - model I - inlay diameter $9.9 \mathrm{~mm}$; (b) bushing insulator — model II inlay diameter $9.9 \mathrm{~mm}$; (c) post insulator - model I; (d) post insulator - model II.

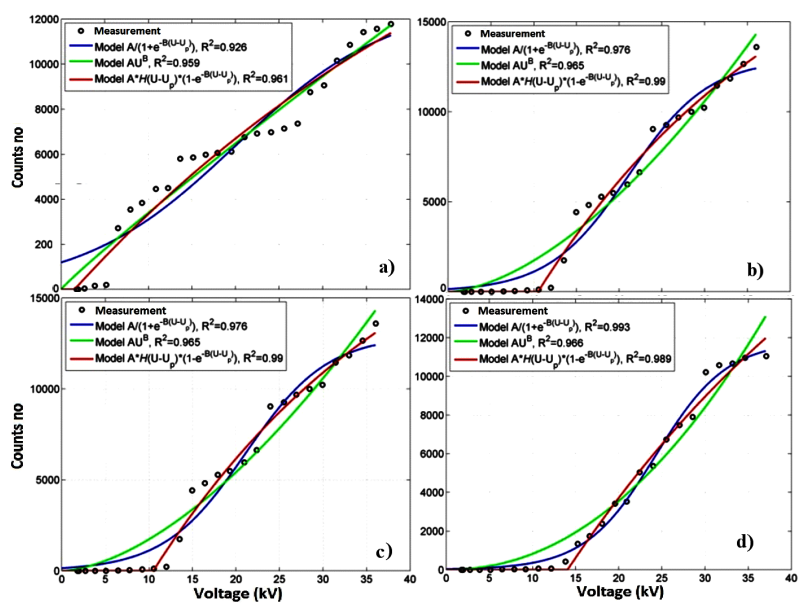

Fig. 3. Change of the number of PD counts, which are determined from measurements, and modelled for the distance of $8 \mathrm{~cm}$ between the electrodes, for: (a) bushing insulator - model I - inlay diameter $9.9 \mathrm{~mm}$, (b) bushing insulator - model II - the inlay's diameter $9.9 \mathrm{~mm}$, (c) post insulator - model I, (d) post insulator - model II. 


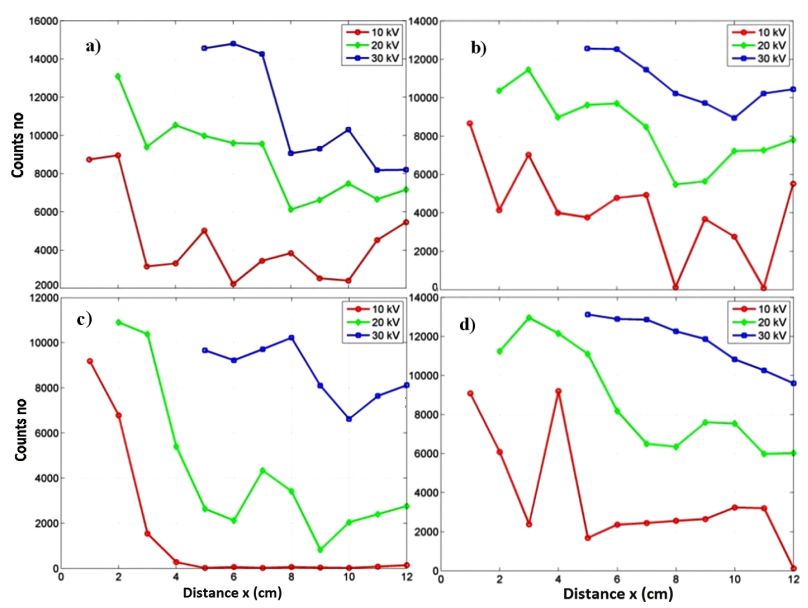

Fig. 4. Correlation between the number of PD counts and the change of the distance between the electrodes, which was determined for three selected values of the voltage $U=10,20$, and $30 \mathrm{kV}$, for: (a) bushing insulator — model I - inlay's diameter $9.9 \mathrm{~mm}$; (b) bushing insulator - model II — inlay's diameter $9.9 \mathrm{~mm}$; (c) post insulator - model I; (d) post insulator - model II.

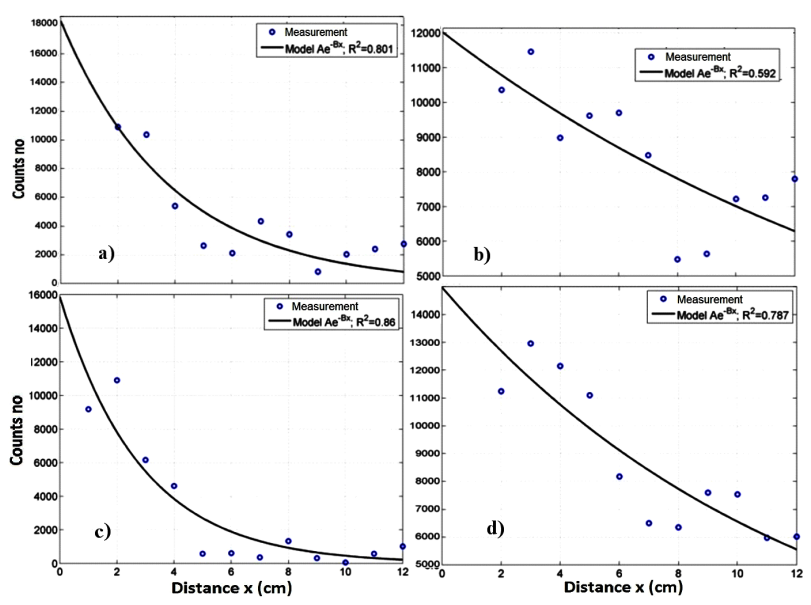

Fig. 5. Correlation between the number of PD counts and the changes of the distance between the electrodes at the voltage $U=20 \mathrm{kV}$, obtained from measurements, and modelled for: (a) bushing insulator - model I inlay's diameter of $9.9 \mathrm{~mm}$; (b) bushing insulator model II - inlay's diameter of $9.9 \mathrm{~mm}$; (c) post insulator - model I; (d) post insulator - model II.

increasing) on the relationships was obtained. Therefore, this article presents characteristics solely obtained when increasing the voltage which supplies the examined models of insulators.

In order to determine the correlation describing the change in the number of PD counts, recorded with the UV camera, and the voltage which supplies the tested insulators, the following three modelling functions were chosen:

- exponential model (two parameters):
$I=A U^{B}$,

- sigmoidal model (three parameters):

$$
I=\frac{A}{1+\mathrm{e}^{-B\left(U-U_{\mathrm{p}}\right)}},
$$

- sigmoidal model with unit step function (Heaviside's):

$$
I=\frac{A}{1+\mathrm{e}^{-B\left(U-U_{\mathrm{p}}\right)}} H\left(U-U_{\mathrm{p}}\right),
$$

where $A, B$, and $U_{\mathrm{p}}$ - constant coefficients of the model, $U$ - value of the voltage supply.

For sigmoidal models, the coefficient $A$ is the amplitude of saturation intensity value, $B$ is the sigmoid slope coefficient, and $U_{\mathrm{p}}$ is the value of voltage at the point of inflection of the curve. For the model with step function, the additional component includes the threshold nature of the radiation emission occurrence (threshold voltage occurrence of PD). The Nelder-Mead minimizing algorithm, being implemented in MATLAB, was used for the approximation.

Figure 3 shows characteristics of correlation between the number of counted PDs and the power supply voltage, in the range from 0 to $0.9 U_{\mathrm{p}}$, which were determined separately for the examined insulators. The graphs shown in Fig. 3 were determined using the measurements, and modelled for the distance between the electrodes, which equals $8 \mathrm{~cm}$. For each of the considered models, coefficients' values and coefficient of determination $R^{2}$ values were calculated separately for each of the tested distances.

For comparison, Fig. 4 illustrates the correlation of the number of PD counts and the distance between the electrodes, which were presented separately for each of the examined models of insulators. The characteristics were determined for three supply voltage values: $U=10,20$, and $30 \mathrm{kV}$.

Figure 5 shows some graphs that illustrate the correlation between the PD counts and distance changes between the electrodes, which were determined separately for the tested insulators, using the same supply voltage of $20 \mathrm{kV}$. The characteristics show the results of measurements (points) and calculated functional correlation between the chosen model (solid line) and the corresponding value of the coefficient of determination $R^{2}$.

\section{Conclusions}

Based on the conducted laboratory tests on recording of the UV radiation emitted by the $\mathrm{PD}$ generated on the tested models of bushing and post insulators, and the analyses of the obtained results, the following conclusions can be made:

- Increase in distance between the electrodes in the range from 1 to $12 \mathrm{~cm}$, regardless of the examined model of the insulator, causes increase in the number of counted PD.

- Based on the comparison of the wave forms of the characteristics showing correlation of the PD counts 
value and changes in distance between the electrodes, examples of which are shown in Fig. 3, can be stated that:

- for all modelling systems of the tested insulators, the increase of supply voltage value, for the given distance between the electrodes in the range from 1 to $12 \mathrm{~cm}$, and fixed values of other parameters set out in point 2 , increases the number of PD counts, where similar shapes of the illustrated wave forms were obtained. In addition, there is a possibility to select approximating functions of the recorded changes, which are characterized by the values of the coefficient of determination above 0.9 , which indicates high adjustment level to each of the three proposed functional models, expressed by Eqs. (1)-(3).

- for the post insulators (model I and II) the ignition of $\mathrm{PD}$ and the active pixels in the UV band counts occur at higher supply voltage values than in the case of bushing insulators modelling systems.

- Entering the reference voltage value $(U=10,20$ and $30 \mathrm{kV}$ ) and comparing the wave forms of the determined characteristics (Fig. 4) it can be stated that for each of the tested insulators for the given distance between the electrodes for higher voltage values, higher number of PD counts were obtained.

- It is possible to approximate the correlation of the number of PD counts for a given voltage and constant values of the parameters associated with the applied UV camera, set for different distances between the electrodes of the insulator, using a function in the form of $A \mathrm{e}^{-B x}$ (example of the graphs in Fig. 5). At the same time, for the considered models of the insulators, different values of the coefficient of determination $R^{2}$, in the range from
0.007 to 0.944 , were obtained. It was caused by different number of measurements taken for a given voltage and distance. In addition, its value that was determined for the insulator modelling system, is variable for the analysed supply voltages.

\section{References}

[1] T. Boczar, S. Borucki, A. Cichon, M. Lorenc, Acta Phys. Pol. A 114, A21 (2008).

[2] S. Borucki, T. Boczar, A. Cichon, Arch. Acoust. 36, 49 (2011).

[3] J. Ignac-Nowicka, T. Pustelny, Z. Opilski, W. Maciak, W. Jakubik, M. Urbanczyk, Opt. Eng. 42, 2978 (2003).

[4] S. Borucki, Acta Phys. Pol. A 120, 571 (2011).

[5] D. Wotzka, T. Boczar, P. Frącz, Acta Phys. Pol. A 120, 767 (2011).

[6] D. Wotzka, A. Cichon, T. Boczar, Arch. Acoust. 37, 19 (2012).

[7] T. Pustelny, I. Zielonka, C. Tyszkiewicz, P. Karasinski, B. Pustelny, Opto-Electron. Rev. 14, 161 (2006).

[8] D. Wotzka, T. Boczar, D. Zmarzły, Acta Phys. Pol. A 118, 1272 (2010).

[9] D. Wotzka, T. Boczar, D. Zmarzły, Acta Phys. Pol. A 116, 428 (2009).

[10] F. Witos, A. Olszewska, Acta Phys. Pol. A 118, 1267 (2010).

[11] P. Frącz, Acta Phys. Pol. A 120, 604 (2011). 